\title{
REVISITING POTENTIAL HAZARD OF LED SOURCES TO CAUSE BLH IN SPECIFIC POPULATION
}

\author{
Pons, A., Campos, J., Ferrero, A., Bris, J.L. \\ Instituto de Óptica "Daza de Valdés" (IO-CSIC), Agencia Estatal CSIC, Madrid, SPAIN \\ alicia.pons@csic.es
}

DOI 10.25039/x44.2017.PO104

\begin{abstract}
Following the strategy in a previous work, we have evaluated the potential Blue-Light Hazard (BLH) of high colour rendering index LED sources, on people whose crystalline lenses have been removed through a surgical procedure to implant intraocular lenses. Starting from the spectral transmittance of various models of commercial intraocular lenses (IOLs), modifications of the action spectrum for the BLH photo biological effect are proposed for each type of IOL. Then, using the corrected action spectra, the potential hazard of different types of white LED sources on subjects implanted with those IOLs, as compared to the potential hazard of the same sources for a person whose crystalline lens has not been removed, has been calculated.
\end{abstract}

Keywords: Blue Light Hazard, Intraocular lenses

\section{Introduction}

The BLH is characterized by its action spectrum (CIE, 2000), which represents the relative weight of each wavelength in terms of the potential damage it can cause to the retina. This permits a direct comparison of different radiation sources to determine the relative effectiveness or the potential hazard that each one can cause. The global BLH for a given source depends, not only on the total radiant power emitted by the source, but also on its relative spectral distribution.

In addition to the function $B(\lambda)$ for the normal eye an additional function $A(\lambda)$ was later developed based on studies of Ham in rhesus monkeys with the crystalline lens surgically removed (Ham, 1982). The function $A(\lambda)$ (the aphake hazard function) should then be applied for persons whose crystalline lens has been removed through a chirurgical procedure or do not have an intraocular lens which absorbs ultraviolet radiant energy in the UV-A spectral region. Figure 1 shows the two different action spectra for $B L H: B(\lambda)$, which represents the action spectrum for a standard (phakic) eye; and $\mathrm{A}(\lambda)$ (aphakic eye) as recommended by $\mathrm{CIE}$.

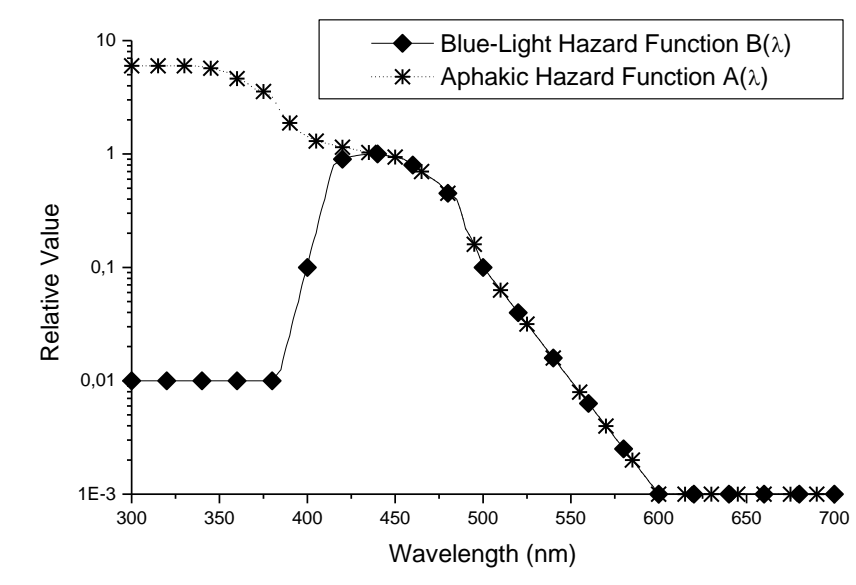

Figure 1 - Spectral weighting functions for retinal hazards $A(\lambda)$ (aphakic eye) and $B(\lambda)$ (normal eye) 
Nowadays there are very few occurrences of aphakic eyes since usually during cataract surgery the removed crystalline lens is replaced by an intraocular lens (IOL). Although, in general, IOLs are provided with optical filtering (UV and blue blocking), they usually transmit much more violet and blue light (even occasionally part of UV) than crystalline lenses at any age, then potential hazard for photochemical injury can be supposed.

In an earlier work on this subject, applied to conventional white LEDs (with blue LED and phosphor technology), which do not deliver short-wavelength radiation, it was concluded that the increase of the hazard factor with respect to a standard eye was non-significant for the studied IOLs.

However, in recent years, we have witnessed a growing use of high colour rendering index LED sources, especially in applications that require better light quality in terms of colour rendering. These types of white LEDs use primarily violet LEDs (and phosphor technology), significantly increasing the emission of radiation at short wavelengths.

This fact, together with the development of new types of intraocular lenses, has led us to evaluate the potential hazard to cause BLH of white LED sources (with violet LED + phosphor technology), on subjects whose crystalline lenses have been removed to implant intraocular lenses.

The action spectrum for the BLH plays the role of spectral weighting function, which has to be included in the calculations of the threshold limit values (TLVs). Based on these values one can then evaluate the potential hazard to cause BLH or the recommended maximum exposure times for a given radiation source. The relative hazard of a given radiation source is defined as follows:

$$
X_{\text {blue }}=\sum_{305}^{700} X_{\lambda} B(\lambda) \Delta \lambda
$$

where $X_{\lambda}$ is the spectral radiance or spectral irradiance of the optical radiation source, $B(\lambda)$ is the BLH function which represents the relative spectral effectiveness for the BLH, and $\Delta \lambda$ is a wavelength interval at the centre of which $B(\lambda)$ is defined and over which spectral irradiance or spectral radiance is measured.

\section{Intraocular lenses}

Six different models of commercial intraocular lenses manufactured by Bausch \& Lomb have been used. Table 1 shows the main features of each IOL. As can be seen, all are provided with a UV filter.

Table 1 - Intraocular Lenses (IOLs) Characteristics

\begin{tabular}{|l|l|l|}
\hline Material & Filter & Name of lens \\
\hline polymethylmethacrylate & UV & L122 UV \\
\hline polymethylmethacrylate & UV & PMMA \\
\hline Silicone & UV & Sofport \\
\hline $\begin{array}{l}\text { Hydrophilic acrylic } \\
\text { copolymer }\end{array}$ & UV & AKREOS \\
\hline Silicone Elastomero & UV & HD500 \\
\hline Hydrophobic Acrylic & UV & EnVista \\
\hline
\end{tabular}

The spectral transmittance for each one of them was experimentally measured by means of a Perkin Elmer spectrophotometer (model Lambda 1050) in the $200 \mathrm{~nm}-700 \mathrm{~nm}$ wavelength interval, with a bandwidth of $0,5 \mathrm{~nm}$ at steps of $1 \mathrm{~nm}$. The IOLs are distributed inside a sterile 
envelope that should be opened only under sterile conditions similar to those found in an operating room to guarantee a complete absence of contamination. However, the sterility conditions should not affect, in principle, the transmittance properties of the lens. The present study has been carried out in a standard laboratory, which doesn't meet the requirements to be considered a sterile one. Two sets of measurements were recorded for each lens under different conditions: immersed in a saline solution and mounted directly on air. Different results regarding absolute values of transmittance have been found between these measurement techniques, that can be explained because of the Fresnel reflection coefficients associated with the air-IOL interface ${ }^{18}$, but no change is observed regarding the cutoff wavelength due to the immersion media, which is the most important aspect for the purpose of this work.

Figure 2 shows the normalized spectral transmittance curves for every intraocular lens, in comparison with ideal transmittance of crystalline lens (calculated as expressed in section 2.1). The normalization has been chosen to obtain transmission unity in the maximum, in order to compare with the ideal transmission of crystalline lens. Significant differences among the various IOLs, mostly regarding the cut-off wavelength, can be seen. They all show a complete absorption of UVC $(200 \mathrm{~nm}-280 \mathrm{~nm})$ and UVB $(280 \mathrm{~nm}-320 \mathrm{~nm})$; however, their behavior inside the UVA range $(320 \mathrm{~nm}-400 \mathrm{~nm})$ differs considerably. Only Sofport completely absorbs UVA radiation, while the rest of the IOLs under analysis show different values for the cut-off wavelength, ranging from $350 \mathrm{~nm}$ (Akreos, HD500 and EnVista) to 370 $\mathrm{nm}$ or $380 \mathrm{~nm}$ (PMMA and L122UV).

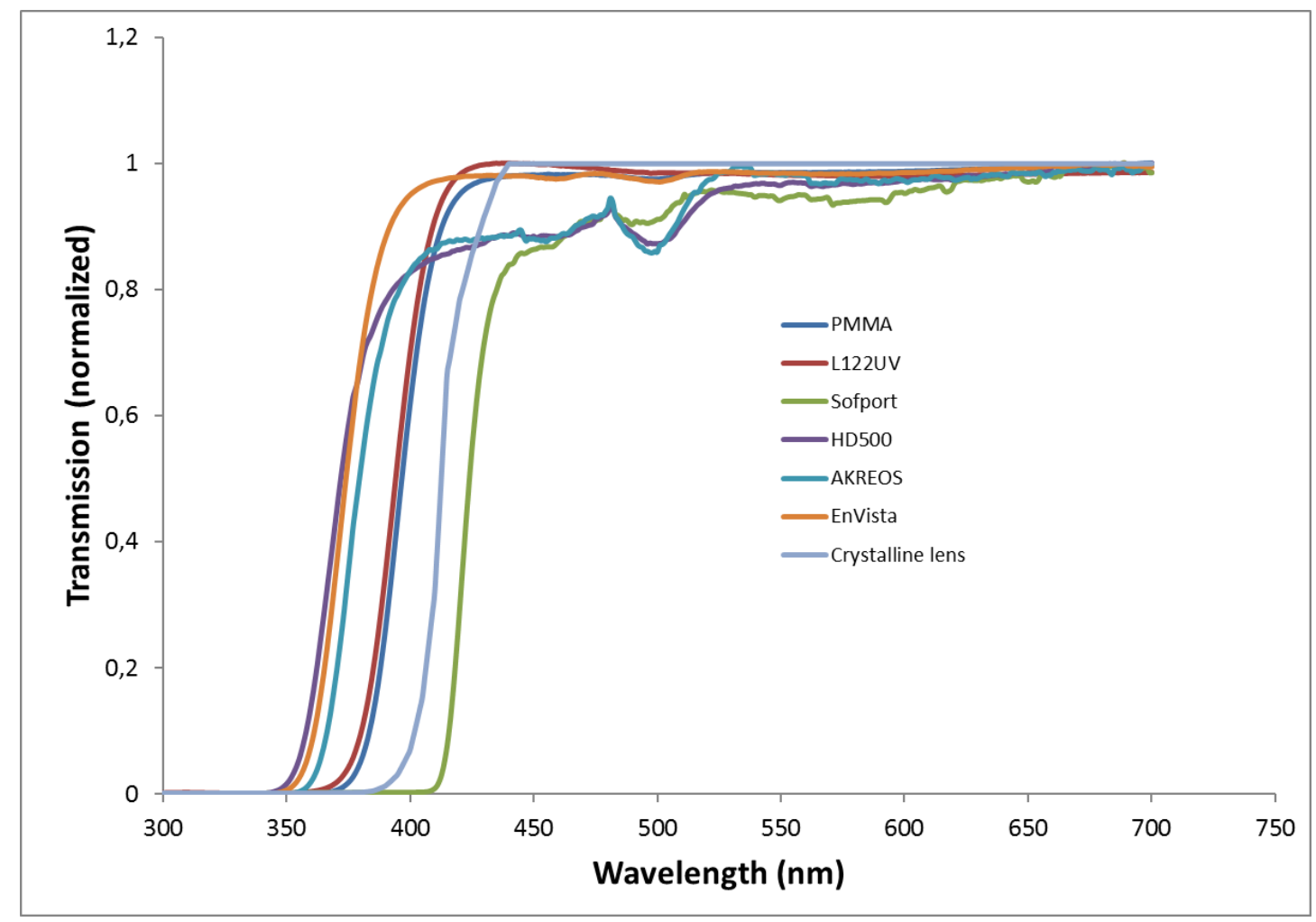

Figure 2 - Transmittance curves (normalized) of the intraocular lenses under study in comparison with the ideal transmittance of crystalline lens

\subsection{Impact upon the action spectrum for the BLH}

Considering that the discrepancy between the two action spectra for the BLH discussed here $(A(\lambda)$ for aphakic eyes and $B(\lambda)$ for standard, phakic eyes) is only due to the effect of the crystalline lens, it means that crystalline's ideal spectral transmittance can be inferred by means of the $B(\lambda) / A(\lambda)$ ratio; that is:

$$
B(\lambda)=\tau_{\text {crystalline }}(\lambda) \cdot A(\lambda)
$$


Then, and just by reversing the above reasoning, it will be possible to calculate the corrected action spectrum function for eyes implanted with IOLs if its spectral transmittance is known. Thus, applying equation (2) to every of the IOLs under study yields.

$$
B_{I O L}(\lambda)=\tau_{I O L}(\lambda) \cdot A(\lambda)
$$

Figure 3 shows the resulting curves $\mathrm{B}_{\mathrm{IOL}}(\lambda)$ for each IOL after applying equation (3) to the spectral transmittances that had been measured.

The differences in transmittance among the different IOLs and with respect to the crystalline lens, especially regarding the cut-off wavelength, imply significant modifications of the corresponding action spectrum in the UVA region. For longer wavelengths, the alterations to the action spectrum are either very small or even non-existent.

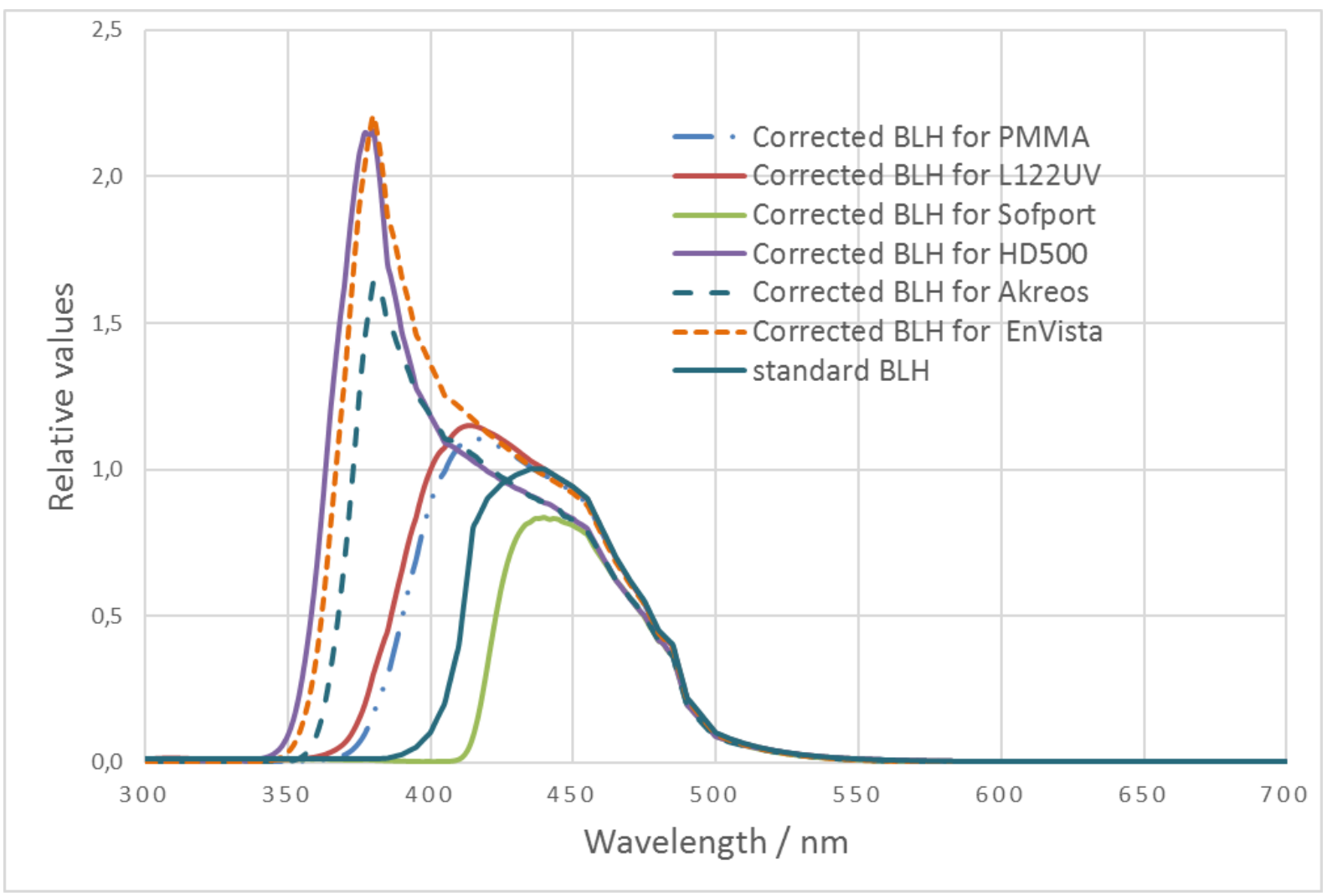

Figure 3 - Standard action spectrum for BLH and corrected action spectra computed for each IOL

\subsection{Assessment of the potential hazard for LED sources}

Replacing in equation (1) the corrected $B(\lambda)$ function (the ones shown in figure 3 ) and including the corresponding relative irradiance curve for the radiation sources under analysis (white LED sources in this work), it is possible to calculate the potential hazard that each source presents to wearers of the different IOLs as compared to the potential hazard of the sources for a person whose crystalline lens has not been extracted.

The LED sources of choice for the present study were the following ones:

Single high power LEDs (with blue LED and phosphor technology): Warm white, White, Cool white and Neutral white manufactured by Cree, Philips and Osram.

Clear non-directional LED lamps (including several LED filament lamps) (with blue LED and phosphor technology): warm white, cool white and neutral white manufactured by different companies. 
High colour rendering index LED sources (with violet LED and phosphor technology): warm white and cool white manufactured by SORAA. In recent years, we have witnessed a growing use of high colour rendering index LED sources, especially in applications that require better light quality in terms of colour rendering. These types of white LEDs use primarily violet LEDs (and phosphor technology), significantly increasing the emission of radiation at short wavelengths.

This study tested a total of 56 LED sources (30 high power single LEDS; 20 clear LED lamps and 6 violet LED lamps). Relative spectral irradiances of LED sources were measured in our laboratory by means of a UV-VIS spectroradiometer.

To evaluate the potential hazard of the different sources we have calculated the Xblue (IOL)/Xblue(BLH standard) ratio obtained for each radiation source under study and for each IOL (i.e., for each corrected action spectrum). Table 2 shows the most representative values obtained for every LED type, identified as: ShpLEDww; ShpLEDw; ShpLEDcw; ShpLEDnw; CLEDlampww; CLEDlampcw; CLEDlampnw; VioletLEDww and VioletLEDcw.

Table 2 - Relative hazard factor $\mathbf{X}_{\text {blue(IOL) }} / \mathbf{X}_{\text {blue(BLH standard) }}$

\begin{tabular}{|l|l|l|l|l|l|l|}
\hline LED source & L122 UV & PMMA & Sofport & AKREOS & HD500 & EnVista \\
\hline ShpLEDww & 1,01 & 0,99 & 0,87 & 0,93 & 0,97 & 1,04 \\
\hline ShpLEDw & 1,03 & 1,01 & 0,83 & 0,93 & 0,95 & 1,04 \\
\hline ShpLEDcw & 1,02 & 1,00 & 0,84 & 0,92 & 0,94 & 1,03 \\
\hline ShpLEDnw & 1,00 & 0,99 & 0,87 & 0,92 & 0,95 & 1,03 \\
\hline CLEDlampww & 1,01 & 0,99 & 0,86 & 0,91 & 0,91 & 0,99 \\
\hline CLEDlampcw & 1,01 & 0,99 & 0,85 & 0,90 & 0,90 & 0,99 \\
\hline CLEDlampnw & 1,01 & 0,99 & 0,85 & 0,90 & 0,90 & 0,99 \\
\hline VioletLEDww & 1,44 & 1,39 & 0,56 & 1,36 & 1,35 & 1,51 \\
\hline VioletLEDcw & 1,34 & 1,30 & 0,66 & 1,26 & 1,25 & 1,40 \\
\hline
\end{tabular}

According to these values, looking at the SSL devices with blue LED technology, nonsignificant increase of the hazard factor with respect to a standard eye can be suggested. However different results have been observed for the SSL devices with violet LED technology. With the exception of the Sofport IOL whose associated hazard factor is between $35 \%$ and 44 $\%$ lower than that for the standard eye, the rest of IOLs induce a significant increase of the hazard factor.

The authors have no interests in the development or marketing of any product mentioned in this study.

\section{References}

CIE 2000 CIE 138:2000. CIE Collection In Photobiology and Photochemistry. Vienna:CIE.

CORREDERA, P. 1991 et al. Realization of an absolute spectroradiometer. Appl Opt, 30, 1279-1284

HAM, W.T.1982 et al. Action spectrum for retinal injury from near-ultraviolet radiation in the aphakic monkey. Am J Ophthalmol, 299-306.

PONS, A. 2007. Determination of the action spectrum of the blue-light hazard for different intraocular lenses. J. Opt.Soc.Am.A,24(6), 1545-1550. 